\title{
Recognizing recurrent neural networks (rRNN): Bayesian inference for recurrent neural networks
}

\author{
Sebastian Bitzer · Stefan J. Kiebel
}

Received: 21 June 2011 / Accepted: 19 April 2012 / Published online: 12 May 2012

(c) The Author(s) 2012. This article is published with open access at Springerlink.com

\begin{abstract}
Recurrent neural networks (RNNs) are widely used in computational neuroscience and machine learning applications. In an RNN, each neuron computes its output as a nonlinear function of its integrated input. While the importance of RNNs, especially as models of brain processing, is undisputed, it is also widely acknowledged that the computations in standard RNN models may be an over-simplification of what real neuronal networks compute. Here, we suggest that the RNN approach may be made computationally more powerful by its fusion with Bayesian inference techniques for nonlinear dynamical systems. In this scheme, we use an RNN as a generative model of dynamic input caused by the environment, e.g. of speech or kinematics. Given this generative RNN model, we derive Bayesian update equations that can decode its output. Critically, these updates define a 'recognizing RNN' (rRNN), in which neurons compute and exchange prediction and prediction error messages. The rRNN has several desirable features that a conventional RNN does not have, e.g. fast decoding of dynamic stimuli and robustness to initial conditions and noise. Furthermore, it implements a predictive coding scheme for dynamic inputs. We suggest that the Bayesian inversion of RNNs may be useful both as a model of brain function and as a machine learning tool. We illustrate the use of the rRNN by an application to the online decoding (i.e. recognition) of human kinematics.
\end{abstract}

Keywords Recurrent neural networks .

Bayesian inference $\cdot$ Nonlinear dynamics $\cdot$ Human motion

S. Bitzer $(\varangle) \cdot$ S. J. Kiebel

MPI for Human Cognitive and Brain Sciences, Stephanstr. 1a, 04107 Leipzig, Germany

e-mail: bitzer@cbs.mpg.de

S. J. Kiebel

e-mail: kiebel@cbs.mpg.de

\section{Introduction}

Recurrent neural networks (RNNs) have been used for many years now to augment nonlinear mappings with a dynamic representation (Pearlmutter 1989; Williams and Zipser 1989; Narendra and Parthasarathy 1990; Jaeger 2001; Maass et al. 2002), e.g. for the classification of sensory input in machine learning. In computational neuroscience, RNNs are extensively used to investigate the dynamic properties of cortical networks (e.g. Buonomano and Maass 2009; Legenstein and Maass 2007), to model the measured activity of networks of neurons (e.g. Friston et al. 2003; Kiebel et al. 2006, 2009a; Sotero et al. 2007; Rodrigues et al. 2010) and more generally to model brain processes like perception, memory and attention (Elman 1990; Miller and Cohen 2001; Hamker 2005). The recurrent connections of these networks capture two of the most prominent features of neuronal networks observed in the brain: first, connections between two neurons are rarely uni-directional but more often bi-directional, potentially via more than one synapse. Second, neurons perform highly nonlinear operations, i.e. they transform their input to spiking output. RNNs capture both these features where often the input (post-synaptic potentials) and output (action potentials) are replaced by summary measures, i.e. the post-membrane potential function and firing rate. In such a continuous-time RNN, each neuron (often called unit) performs a simple operation: in each moment in time, it applies a nonlinear function to the sum of its input and passes this on to other neurons. This simple mechanism can provide for extremely rich patterns of activity in each neuron, even with a network of small size. Literally, thousands of contributions in computational neuroscience and machine learning are based on networks of these firing ratecoding units (Rabinovich et al. 2006; Cessac and Samuelides 2007). 
As powerful as RNNs are as a model class, they are still a rather simple abstraction from what is known about real neuronal networks. For example, it has been suggested that even single neurons may compute more complex functions of their input than single units in an RNN (Poirazi et al. 2003; Spruston 2008; Mel 2008; Debanne et al. 2011). Here, we suggest that a simple re-interpretation of the functional role of RNN dynamics leads to a novel and potentially more plausible account of what recurrently connected units may compute: we suggest that neuronal networks serve as Bayesian decoders of dynamics caused by the environment. For example, in action observation, humans decode the kinematics of other people from visual input dynamics. Bayesian recognition is based on a so-called generative model which is an internal representation of the hidden dynamics of the environment which cause sensory input to the brain. We suggest that RNNs are an ideal generative model for these hidden dynamics in our environment. The task of the recognition system is to decode the sensory input generated by the hidden RNN dynamics. To do this, we derive Bayesian update equations from the generative RNN model and call these 'recognizing RNN' (rRNN). As a consequence, there is a major difference between the rRNN and the standard RNNs used for discriminating dynamic input: while standard RNNs represent features specialized for the task of discrimination, the rRNN maintains a full representation of the input through the generative RNN. In particular, the rRNN can predict the future evolution of the input and may, therefore, also be useful in tasks other than the pure recognition task considered here. Technically, the difference to a standard RNN is that each unit in the rRNN computes more sophisticated updates involving predictions and prediction error messages from other units in the network. Here, we show that a rRNN can decode real-world dynamics (human kinematics) and can display several features which can also be observed with real neuronal systems, e.g. the online decoding of hidden dynamics in the environment, computation of predictions and prediction error, robustness to noisy input and fast adaptation to sudden changes in the environment. These features are not only general hallmarks of brain function but, in principle, also may be useful for machine learning applications for decoding dynamics in an online fashion.

In computational neuroscience, models of recurrently connected networks of neurons, which optimally estimate dynamically changing states from noisy observations, have recently been proposed (Rao 2004; Denève et al. 2007; Natarajan et al. 2008; Wilson and Finkel 2009; Boerlin and Denève 2011). While these models provide important insights, results were reported for relatively restrictive conditions such as linear dynamics (Denève et al. 2007; Wilson and Finkel 2009; Boerlin and Denève 2011), discrete states (Rao 2004; Denève et al. 2007; Boerlin and Denève 2011), or a one-dimensional state (Natarajan et al. 2008; Wilson and
Finkel 2009; Boerlin and Denève 2011). Although, Natarajan et al. (2008) allow for nonlinear dynamics they assume knowledge of an ideal observer which provides an instantaneous error signal for learning of network connections. Similarly, reservoir computing approaches (Jaeger 2001; Maass et al. 2002; Verstraeten et al. 2007) rely on a teaching signal which provides a desired output at every point in time during learning. In contrast, we propose an approach combining multi-dimensional, continuous-time hidden nonlinear dynamics where learning proceeds without an externally provided error signal. Our main contribution is to demonstrate that a rRNN is well suited to recognize dynamic stimuli and may be used as a functional model for neuronal ensemble dynamics. In particular, we will illustrate this by showing that the prediction errors computed by a rRNN provide sufficient information to discriminate dynamic stimuli, in an online fashion.

The present approach may also lead to a better understanding of the role of recurrently connected networks of neurons in the brain: predictive coding has been suggested as a theory for hierarchical processing in the brain in which different levels exchange prediction and prediction error messages (Mumford 1996; Rao and Ballard 1997, 1999; Friston and Kiebel 2009). Rao and Ballard (1997) already described RNN-like dynamic models to implement predictive coding for static stimuli. The present approach can be seen as an extension to Rao and Ballard's original work to provide inference for dynamic stimuli by resorting to approximate inference methods for nonlinear, continuous dynamic models (Friston et al. 2008).

The remainder of the paper is organized as follows. In Sect.2, we (i) present RNNs as generative models, (ii) describe the Bayesian inference framework and (iii) show that dynamic updates of the posterior state critically depend on prediction error. We illustrate the rRNN approach using human motion capture data. In Sect. 3, we demonstrate that the rRNN can successfully recognize human kinematics and discriminate between different walking styles based on the prediction error of rRNN units.

\section{Materials and methods}

In the following, we will describe the two key elements of the present approach: a RNN as a generative model of the sensory dynamics and the Bayesian inference framework to derive the update equations for a rRNN. Subsequently, we will apply the rRNN technique to the recognition of human kinematics, for which we describe the kinematic data and the rRNN settings.

To motivate the present approach, we will start with a brief summary of the conventional RNN technique as used in machine learning for classification of stimuli. Note, however, 
that it is not our aim to compare discrimination performance of conventional and rRNNs. Rather, description of the conventional RNN is given as a reference for understanding the conceptual differences between the two approaches.

\subsection{Conventional RNN}

The RNN technique has been used in many machine learning applications such as classification of static or dynamic stimuli, or time-series prediction. This approach has a long history which took off with the development of a supervised learning routine (Pearlmutter 1989; Williams and Zipser 1989). Recently, this learning approach has been complemented by the so-called reservoir computing technique (Jaeger 2001; Maass et al. 2002).

In general, in a conventional $\mathrm{RNN}$, sensory units provide input, which drives the dynamics of the hidden units (see Fig. 1a). Output units readout the result of the dynamic computations based on a mixing of the sensory and hidden states.

Typically, RNNs come in two different types: either as networks of spiking neurons, typically modelled as leakyintegrate-and-fire neurons, or as networks of more abstract neuronal units which model summary measures of neuronal spiking such as the firing rate. Here, we consider the latter type of RNNs with leaky-integrator units which, for the application considered here, have the advantage over spiking neuron models that the states of these units change continuously over time and are not subject to discontinuous jumps introduced by the spiking mechanism. An example of such a network is discussed in Jaeger et al. (2007) where the continuous-time dynamics based on leaky-integrator units is given by

$$
\begin{aligned}
\dot{x}_{i} & =f(\mathbf{x})_{i} \\
& =k_{i}\left(-a x_{i}+\tanh \left(\left[\mathbf{W}^{\mathrm{in}} \mathbf{y}+\mathbf{W} \mathbf{x}+\mathbf{W}^{\mathrm{fb}} \mathbf{o}\right]_{i}\right)\right),
\end{aligned}
$$

where $x_{i}$ is the state of hidden unit $i \in\{1, \ldots, H\}, \mathbf{y} \in \mathbb{R}^{I \times 1}$ are the states of the input (sensory) units, $\mathbf{o} \in \mathbb{R}^{D \times 1}$ are the states of the readout units, $\mathbf{W} \in \mathbb{R}^{H \times H}$ is a weight matrix defining the interaction between the $H$ hidden units, similarly $\mathbf{W}^{\text {in }}$ and $\mathbf{W}^{\mathrm{fb}}$ define the connections from the input to the hidden units and the (optional) feedback connections from the readout units, respectively. $k_{i}$ is a rate constant for unit $i$ and $a$ is the amount of leakage. The output states are determined by

$\mathbf{o}=\mathbf{V}\left[\mathbf{x}^{T}, \mathbf{y}^{T}\right]^{T}$

where $\mathbf{V} \in \mathbb{R}^{D \times(H+I)}$ is a weight matrix.

In a conventional RNN, the overall flow of information is from sensory to output units, because the RNN serves as a model for neuronal dynamics (hidden states) which are used to compute, e.g. a classification of the sensory input. We now use the same dynamics where we reverse the flow of informa- tion to model the generation of sensory dynamics by hidden states of the environment (e.g. body movements cause visual output dynamics).

\subsection{Generative RNN}

Our overall aim is to construct a recognition system which can recognize its sensory observations based on its internal dynamics. For a Bayesian recognition system we require a dynamic generative model, for which we choose a RNN. This 'generative recurrent neural network' (gRNN) runs independently of any input and generates sensory data, i.e. observations. Note that, in comparison to a conventional RNN (Eq. 1 ), here the sensory units become the output of the network while no input units are defined (hence the missing units which acted as output in the conventional RNN). Consequently, the flow of information is reversed in the gRNN and its autonomously running hidden dynamics drive its sensory units (see Fig. 1b). In particular, we define a gRNN as

$\dot{x}_{i}=f(\mathbf{x})_{i}=k_{i}\left(-a x_{i}+\tanh \left([\mathbf{W} \mathbf{x}]_{i}\right)\right)$,

$\mathbf{y}=\mathbf{V} \mathbf{x}$

where now $\mathbf{V} \in \mathbb{R}^{D \times H}$ linearly translates hidden states $\mathbf{x}$ into sensory states $\mathbf{y}$. This gRNN computes sensory output $\mathbf{y}$ as caused by a hidden, dynamic process defined by the RNN dynamics $f(\mathbf{x})$. In the following section, we describe how a rRNN is constructed from the gRNN using Bayesian inversion. This rRNN receives sensory observations (as in a conventional RNN, Eq. 1) and infers about the hidden states that caused these observations. Effectively, conventional RNN computations are aimed at doing the same (c.f. Fig. 1a, c); however, the update equations of an rRNN are explicitly derived for this recognition task.

\subsection{Recognizing RNN}

Generative models like a gRNN (Eqs. 3, 4) can be used as the basis for inferring the state of the hidden dynamics given observations which are caused by the generative model. In realistic settings, where observations and state transitions are noisy, or uncertain, inversion of the nonlinear generative model is an ill-defined problem and further assumptions about the hidden states have to be made to disambiguate their possible values. We transform the gRNN, as presented above, into a probabilistic model by adding assumptions about the distributions of hidden states and observations (cf. Eqs. 7, 8). Given the probabilistic generative model (and prior assumptions about the initial hidden states) Bayesian inference is the optimal method to invert the generative model and leads to updates of the hidden states which make them an optimal representation of the observations. For example, the wellknown Kalman-Bucy filter (Jazwinski 1970) implements 


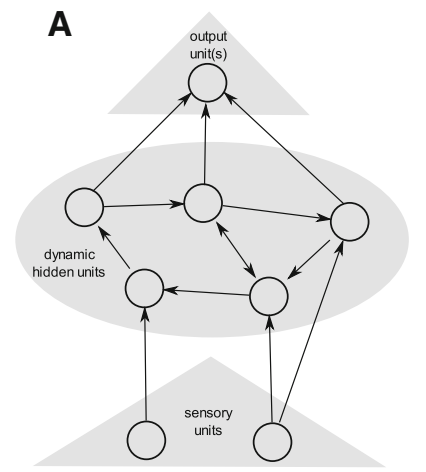

B

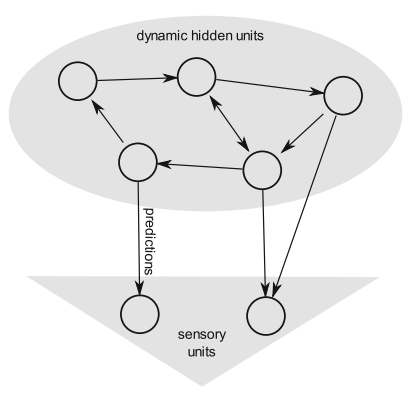

C

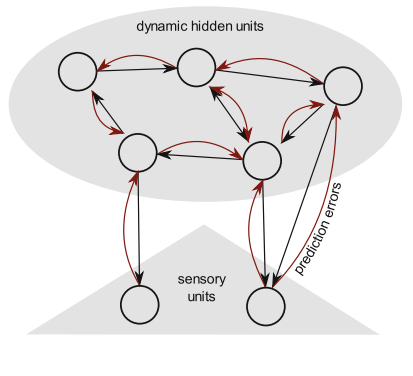

Fig. 1 Comparison of different RNN architectures. a conventional RNN, b generative RNN (gRNN) and $\mathbf{c}$ rRNN. Each RNN has dynamic hidden units, but the overall direction of information flow differs (indicated by the grey triangles). The conventional RNN is designed to com- pute an output given sensory input. In contrast, the gRNN computes sensory states. Finally, the rRNN computes predictions (black arrows) and prediction error messages (red arrows) to recognize the hidden causes that generated the sensory input. (Color figure online) such a Bayesian inversion scheme for linear dynamic processes. The gRNN uses highly nonlinear dynamics (Eq. 3) and, therefore, we require approximate or sampling-based inversion schemes (Jazwinski 1970; Wan and van der Merwe 2001; Doucet et al. 2001; Friston et al. 2008; Daunizeau et al. 2009; Friston et al. 2010). Here, we derived the update equations using the D-step of Friston's dynamic expectation maximization (DEM) framework (Friston et al. 2008). This choice was based on our previous experience with inversion of continuous-time dynamic models using DEM (Kiebel et al. 2009a). In principle, other inversion schemes could be used as well. The advantage of using DEM, or similar schemes like Bayesian inference using discretized dynamics (Daunizeau et al. 2009), is that prediction errors on the dynamics are computed. DEM uses generalized coordinates, local linearization and point-estimates at strategically important positions. See the 'Appendix' for a high-level derivation of the algorithm and an explanation of generalized coordinates which are a dynamically extended representation of state variables, the use of which we indicate by a tilde in the subsequent formulas.

In the following, we will briefly describe the key computations performed by DEM. This description is aimed at giving an intuitive description of the update equations governing the rRNN and will allow interpretation of these updates in terms of prediction and prediction error messages.

The most important equation resulting from inversion with DEM describes the evolution of the posterior mode of the hidden states in generalized coordinates and is given by

$\dot{\tilde{\mathbf{x}}}=\kappa \frac{\partial V(\tilde{\mathbf{x}})}{\partial \tilde{\mathbf{x}}}+\mathbf{D} \tilde{\mathbf{x}}$.

The motion defined in this equation consists of two parts: (1) D $\tilde{\mathbf{x}}$ which, in absence of other contributions, implements that the motion of the posterior mode follows its local trajectory represented in generalized coordinates using a derivative operator $\mathbf{D}$ and (2) the derivative of the variational energy $V(\tilde{\mathbf{x}})$ with respect to hidden states which acts as a corrective force to make the motion consistent with the gRNN and the observations. With fixed parameters, the variational energy is the log-joint probability of observations (sensory states) $\tilde{\mathbf{y}}$ and hidden states $\tilde{\mathbf{x}}$ which defines the probabilistic gRNN. In particular, the variational energy is given by

$$
\begin{aligned}
V(\tilde{\mathbf{x}}) & =\log p(\tilde{\mathbf{y}}, \tilde{\mathbf{x}} \mid \theta) \\
& =\log p(\tilde{\mathbf{y}} \mid \tilde{\mathbf{x}}, \theta)+\log p(\mathbf{D} \tilde{\mathbf{x}} \mid \tilde{\mathbf{f}}, \theta) \\
& =\log p(\tilde{\mathbf{x}} \mid \tilde{\mathbf{y}}, \theta)+c,
\end{aligned}
$$

where $c$ is a constant, $\theta$ is a vector consisting of all parameters of the model and $\tilde{\mathbf{f}}$ are the dynamic predictions defined by Eq. 3 in generalized coordinates. The last term illustrates that the updates are a dynamic form of maximum a posteriori estimation of hidden states. Gaussian distributions are assumed for the state transition and observation densities:

$p(\mathbf{D} \tilde{\mathbf{x}} \mid \tilde{\mathbf{f}}, \theta) \sim \mathcal{N}\left(\tilde{\mathbf{f}}, \tilde{\Sigma}_{x}\right)$,

$p(\tilde{\mathbf{y}} \mid \tilde{\mathbf{x}}, \theta) \sim \mathcal{N}\left((\mathbf{I} \otimes \mathbf{V}) \tilde{\mathbf{x}}, \tilde{\Sigma}_{y}\right)$,

where $(\mathbf{I} \otimes \mathbf{V}) \tilde{\mathbf{x}}$ is the predicted sensory state given the hidden states as defined by Eq. 4 in generalized coordinates, ${ }^{1}$ and $\tilde{\Sigma}_{x}$ and $\tilde{\Sigma}_{y}$ are the prior covariances of sensory and hidden states in generalized coordinates, respectively. This leads to a simple interpretation of the posterior mode updates in terms of prediction errors. In particular, the gradients of these densities with respect to hidden states become

$$
\begin{aligned}
\frac{\partial \log p(\mathbf{D} \tilde{\mathbf{x}} \tilde{\mathbf{f}}, \theta)}{\partial \tilde{\mathbf{x}}} & =-\frac{1}{2} \frac{\partial}{\partial \tilde{\mathbf{x}}} \tilde{\varepsilon}_{x}^{T} \tilde{\Sigma}_{x}^{-1} \tilde{\varepsilon}_{x} \\
& =-\left[\frac{\partial \tilde{\varepsilon}_{x}}{\partial \tilde{\mathbf{x}}}\right]^{T} \tilde{\Sigma}_{x}^{-1} \tilde{\varepsilon}_{x},
\end{aligned}
$$

\footnotetext{
${ }^{1} \mathbf{I}$ is the identity matrix with size equal to the number of used generalized coordinates and $\otimes$ is the Kronecker product.
} 


$$
\begin{aligned}
\frac{\partial \log p(\tilde{\mathbf{y}} \mid \tilde{\mathbf{x}}, \theta)}{\partial \tilde{\mathbf{x}}} & =-\frac{1}{2} \frac{\partial}{\partial \tilde{\mathbf{x}}} \tilde{\varepsilon}_{y}^{T} \tilde{\Sigma}_{y}^{-1} \tilde{\varepsilon}_{y} \\
& =-\left[\frac{\partial \tilde{\varepsilon}_{y}}{\partial \tilde{\mathbf{x}}^{T}}\right]^{T} \tilde{\Sigma}_{y}^{-1} \tilde{\varepsilon}_{y}
\end{aligned}
$$

where the prediction errors are defined as

$\tilde{\varepsilon}_{x}=\mathbf{D} \tilde{\mathbf{x}}-\tilde{\mathbf{f}}$

$\tilde{\varepsilon}_{y}=\tilde{\mathbf{y}}-(\mathbf{I} \otimes \mathbf{V}) \tilde{\mathbf{x}}$.

This means that the updates of the posterior hidden states follow the gradient of the prediction error with step sizes determined by the prediction error itself weighted by the prior precisions. The contribution from the prediction error on the sensory states, $\tilde{\varepsilon}_{y}$, ensures that the sensory states are well explained by the hidden states while the contribution from the prediction error on the hidden states, $\tilde{\varepsilon}_{x}$, ensures that the posterior dynamics of hidden states as encoded by the generalized coordinates is consistent with the learnt model dynamics. In particular, for the first generalized coordinate, the prediction error

$\varepsilon_{x}=\dot{\mathbf{x}}-f(\mathbf{x})$

ensures that the posterior velocity corresponds to the learnt, noise-free hidden unit dynamics as defined in Eq. 3. Conversely, we will argue below that a consistently large prediction error $\varepsilon_{x}$ provides evidence for an inconsistency between observed and learnt dynamics and can be used to discriminate among different dynamic stimuli.

The question remains how the system got to know a suitable gRNN which generates specific sensory dynamics. In our experiments, we let the system learn its generative model by adapting connectivity parameters $\mathbf{W}, \mathbf{V}$ and rate constants $\mathbf{k}$ using an approach which was developed for the identification of dynamical (neural-mass) systems (Friston et al. 2003; Kiebel et al. 2009a) and is based on maximum a posteriori estimation of the parameters (Friston 2002; Friston et al. 2002). See the 'Appendix' for details. Note that this initial learning step is not our main point in this paper; not only any learning approach that successfully learns hidden gRNN dynamics to represent a given dynamic stimulus could be used here (e.g. Wan and Nelson 2001; Roweis and Ghahramani 2001; Valpola and Karhunen 2002; Doucet and Tadić 2003; Archambeau et al. 2008; Friston et al. 2008; Daunizeau et al. 2009; Kantas et al. 2009; Lazar et al. 2009; Schön et al. 2011) but also standard RNN learning may be used, if the hidden state dynamics is assumed to be deterministic during learning.

\subsubsection{Message passing in the rRNN}

The updates defined by Eqs. 5, 9, 10 and 11 can be interpreted as network dynamics based on messages sent by sensory and hidden units. Algebraically, this can be seen by exemplarily inspecting the observation density update equation, Eq. 10, for the first generalized coordinate of a single hidden unit $i$

$$
\begin{aligned}
\frac{\partial \log p(\tilde{\mathbf{y}} \mid \tilde{\mathbf{x}}, \theta)}{\partial x_{i}} & =-\frac{\partial \tilde{\varepsilon}_{y}^{T}}{\partial x_{i}} \tilde{\Sigma}_{y}^{-1} \tilde{\varepsilon}_{y} \\
& =-\sum_{j} \frac{\partial\left[\tilde{\varepsilon}_{y}\right]_{j}}{\partial x_{i}}\left[\tilde{\Sigma}_{y}^{-1} \tilde{\varepsilon}_{y}\right]_{j},
\end{aligned}
$$

where the sum over $j$ runs over sensory units $y_{j}$ in generalized coordinates. Note that the partial derivative of the prediction error of sensory unit $j$ with respect to the state of hidden unit $i$ describes how a change in the state of unit $i$ affects the prediction error of unit $j$. Therefore, the state update for hidden unit $i$ is a weighted sum of these prediction error gradients where each element of this sum corresponds to a 'prediction error message' from a single sensory unit $j$. To compute the prediction error message a sensory unit first has to compute a prediction. This is done using the forward equation (4) of the gRNN which is a weighted sum of the hidden states $\tilde{\mathbf{x}}$ where the weights are determined by the connectivity of the gRNN. In the following, we call the elements of this sum 'prediction messages' which are sent from a hidden unit $x_{i}$ to a sensory unit $y_{j}$. In summary, the update equations define a rRNN where a hidden unit sends prediction messages to connected sensory and hidden units such that these can compute prediction error messages which are returned to the hidden unit to update its state (see also Fig. 1c). The updates resulting from the dynamics density, Eq. 9, follow the same logic, where the hidden unit $x_{j}$ takes the place of sensory unit $y_{j}$. Each hidden unit, therefore, sends and receives two kinds of messages: prediction and prediction error messages.

\subsubsection{Induced connectivity of the $r R N N$}

The connectivity matrices $W$ and $V$ of the gRNN (Eq. 3, 4) are not necessarily the same as in the rRNN. In general, the rRNN will have all connections of the gRNN plus the corresponding reciprocal connections, plus some additional ones. To see this, note that the prediction error messages in the rRNN in Eq. 13 are 0, when hidden unit $i$ is not connected to sensory unit $j$, i.e. when hidden unit $i$ has no direct influence on the computation of predictions in sensory unit $j$ (then $\frac{\partial\left[\tilde{\varepsilon}_{y}\right]_{j}}{\partial x_{i}}=0$ ). Only sensory units which receive a connection from a hidden unit $i$ in the gRNN will contribute messages containing the derivative of the prediction error. However, in the rRNN, sensory units $j$, which are not connected in the gRNN to a hidden unit $i$, may also contribute messages, containing only their prediction error, through the weights computation $w_{j}=\left[\tilde{\Sigma}_{y}^{-1} \tilde{\varepsilon}_{y}\right]_{j}$. In particular, if the $j$ th row of the sensory precision matrix, $\tilde{\Sigma}_{y}^{-1}$, has nonzero entries in positions other than $j$, e.g. $k$, the weight of sensory unit $j$ in the update equation (Eq. 13) depends on the prediction error of unit $k$. In this case, sensory unit $k$ contributes to the update 


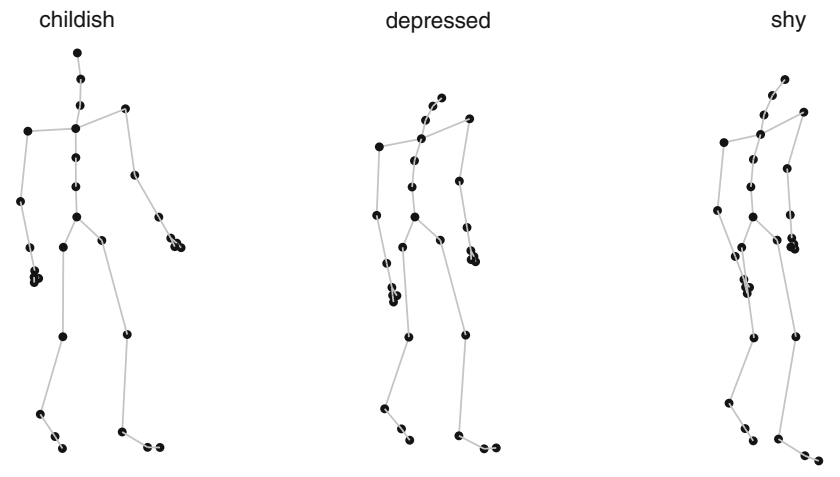

Fig. 2 Example frames from the three different walking styles: 'childish', 'depressed' and 'shy' (left to right). In our experiments we used the first five principal component coordinates of the motion capture 3D joint coordinates (indicated as filled circles) as observation variables. Lines are plotted only for visualization purposes

of hidden unit $i$, even though hidden unit $i$ is not connected to sensory unit $k$ in the gRNN. This means, that there is an additional connection from sensory unit $k$ to hidden unit $i$ in the rRNN.

In conclusion, only if the covariance matrix $\tilde{\Sigma}_{y}$ is diagonal, the connectivity matrix of sensory to hidden units in the rRNN will only contain those connections which are reciprocal to the hidden to sensory unit connections in the gRNN. Conversely, if there are off-diagonal entries in $\tilde{\Sigma}_{y}$, there will be corresponding additional connections from sensory to hidden units in the rRNN, relative to the gRNN. The same considerations apply to the connectivity between hidden units. In summary, the connectivity of the rRNN directly follows from the gRNN, only if the units' states in the gRNN are a priori independent. For simplicity, this case is shown in Fig. 1c and used in the following simulations. Note that a diagonal covariance matrix $\tilde{\Sigma}_{y}$ is a natural assumption for the present data because we assume that the measurement noise is white and any correlation among observations is caused by the underlying dynamics which are modelled by the RNN dynamics.

\subsection{Human movement data}

We use human movements to demonstrate the properties of the rRNN in the experiments below. The kinematics of humans is highly dynamic and nonlinear through complicated interactions between individual joints. Kinematics, therefore, provides a good example of the kind of complex, dynamically changing, real-world stimuli which can be modelled using rRNNs. Here, we used three walks of the same subject, each of which expresses a different walking style (categorized as 'childish', 'depressed' and 'shy'; freely available from the CMU motion capture database, http://mocap. cs.cmu.edu, subject 142, motions 1, 5 and 19). We chose this particular subject because a large range of different move- ments were available among which we chose the selected walks because of their similar time-scales. The advantage of using motion capture data as compared to video is that we can focus on modelling the kinematics of the subject in terms of changing joint angles without the need to model detailed processing of visual information.

For each walk, we removed the global translation of the body. This operation is motivated by the fact that the global translation of the body is merely an effect of the movement executed by the actor, i.e. the actor controls his joints which leads to a feet-ground interaction and moves him forward. Therefore, the relative motion of the joints are the critical features describing a particular movement, as opposed to the body interaction with the static ground. It is these dynamical and complex features which we focus on in the following.

Subsequently, we computed the 3D positions of the joints and extremities for all time points. This removed potential 'jumps' introduced by the circularity of the joint angles. As a result, we obtained a set of 30 points moving in $3 \mathrm{D}$ space (see Fig. 2, for an example). Subsequently, we selected four representative seconds of data starting when the left foot touched the ground for each walk and subsampled the data using 30 frames per second resulting in $N=120$ data points per walk. These data covered roughly two footsteps for each movement. We then found a common, low-dimensional representation for the three walks using principal component analysis (of all the three walks combined) which reduced the dimensionality from 90 dimensions to $D=5$ (maintaining $95.5 \%$ of the original variance). Additionally, we scaled the coordinates of each walk such that the maximum absolute value in each dimension was 1 over all walks. In summary, we obtained for each of the three walks a sequential data set containing five trajectories each consisting of 120 time points, see Fig. 3 .

\subsubsection{Learning of generative RNNS}

For each of the three walks, we constructed one gRNN by learning suitable parameters $\mathbf{W}, \mathbf{V}$ and $\mathbf{k}$ (Eqs. 3, 4) so that the dynamics of each generative RNN replicated the movement data. Each RNN had five sensory units, each of which generated one of the scaled principal component coordinates. In initial tests, we found that a network with $H=12$ hidden units was the smallest network which gave consistently acceptable learning results and we consequently used this network size in our experiments. These tests also showed that good learning results were obtained, if the hidden units were sparsely connected. In particular, we fixed 2/3 of all connections in $\mathbf{W}$ and $1 / 3$ of all connections in $\mathbf{V}$ to 0 . Other entries in $\mathbf{W}$, the rate constants $\mathbf{k}$ and the initial vector of hidden unit states $\mathbf{x}(0)^{l}$ were chosen randomly before learning, while $\mathbf{V}$ was initially chosen to correctly predict the first data point of a walk given $\mathbf{x}(0)^{l}$. For details of this initiali- 
zation and the learning procedure see the 'Appendix'. Note that any learning procedure could have been used here. The main point made by this initial learning step is that a dynamic representation for each walk can be found using RNNs with few units.

The sensory state trajectories of the learnt gRNNs are shown in Fig. 3. Each of the three different walks was learnt well: the amount of variance explained for each walk was 99, 97 and $97 \%$ for the childish, depressed and shy walks, respectively.

\section{Results}

Here, we demonstrate the utility of Bayesian inference for RNNs for online recognition of dynamic stimuli. As a proof of principle, we apply the approach to the multi-dimensional, nonlinear kinematics of a walking human. We will first show that rRNNs quickly and successfully recognize the hidden dynamics, i.e. decode the type of movement. Then, we will demonstrate that the prediction errors of the hidden units can be used to discriminate the three different walks. Finally, we will show that the rRNNs are robust against noisy observations and initial conditions. Note that all the following experiments with the rRNN use the original motion capture data as observations.

\subsection{Fast recognition of dynamics}

In this section, we show that the rRNN can quickly start recognizing a movement online. In particular, we show that this 'quick response' is robust against the initial hidden states at the beginning of the recognition process. This robustness is obtained despite the fact that gRNNs have a large dependence on their internal initial conditions. This is because RNNs are in general rich dynamic models which are capable of simultaneously representing many different dynamic stimuli depending on their initial conditions (hidden states). We demonstrate this for the gRNN for the childish walk. This gRNN was initialized during learning with the state $\mathbf{x}(0)^{l}$. When this specific gRNN is started, after learning, in this state, the learnt shy walk is generated as shown in Fig. 3, left. However, when we initialize the same gRNN with a state $\mathbf{x}(0)^{r}=\mathbf{x}(0)^{l}+\epsilon$, which was perturbed by noise of the same size as the natural variability of the hidden states, it generated very different trajectories of sensory states $\mathbf{y}$ as well as hidden states $\mathbf{x}$ as shown in Fig. 4. In other words, for deviating starting conditions, the gRNN generates dynamics that look different from the learnt kinematics and, when plotted in motion capture space, can deviate severely from a natural walk.

It may be possible to extend the gRNN such that it generates the learnt trajectory independent of initial conditions.
However, our point here is that this is unnecessary, because the rRNN has already this built-in property of robustness against perturbations in the initial states. In particular, the rRNN based on this gRNN for the shy walk was robust against such differences in initial conditions. Even though we perturbed the rRNN initial states severely, the rRNN always switched rapidly to the appropriate dynamics which best described the sensory input of a shy walk. In other words, the prediction error updates of the hidden units forced the dynamics on a trajectory which predicted the observed walk. We depict a characteristic example of this quick response behaviour for the rRNN (childish walk) in Fig. 5a, b. After only one time step the rRNN accurately predicted all subsequent observations, while hidden unit trajectories followed those typical for the learnt gRNN to a large extent (note that these results partially depend on an appropriate choice of the prior covariances, see 'Appendix C'). Although, the perturbation of the hidden states occurs at the beginning of the stream of observations, the same behaviour would be seen, if observations themselves are temporarily perturbed during recognition. This means that the rRNN can represent the dynamic repertoire of the gRNN but, in addition, can rapidly switch to the specific dynamic regime that best explains the sensory input, also after a perturbation.

\subsection{Discrimination of dynamic stimuli}

After learning, we have three different rRNNs, each of which has learnt to predict one of the three walking styles childish, depressed and shy. Here, we will show that the prediction error, on observations $\varepsilon_{y}=\mathbf{y}-\mathbf{V x}$ or hidden states $\varepsilon_{x}=\dot{\mathbf{x}}-f(\mathbf{x})$ (Eq. 12), of all the three rRNNs can be used to discriminate between the three different walks. In particular, we will show that the dynamic prediction errors $\varepsilon_{x}$ are smallest for the rRNN that has learnt a specific walking style. This means that a potential readout mechanism can use the relative amplitudes of prediction error of the three rRNNs to decide which of the three walks is currently observed.

Figure $5 \mathrm{~d}-\mathrm{i}$ shows the response of the rRNN which learnt the childish walk, but now given the depressed and shy walks as observations. Although, this rRNN did not learn these walks it represented them well by exploiting alternative dynamics embedded in the 12 unit network. However, the rRNN frequently had to use prediction error on its hidden states to explain away the remaining mismatch between internal predictions and actual input. See Fig. 5c, f, i for this relative increase in prediction error in response to the nonlearnt depressed and shy walks. This increase in prediction error when recognizing the two nonlearnt walks is consistent over the three different rRNNs and may be used to discriminate dynamic stimuli as shown in Fig. 6d-f. For each of the three walks the prediction error was smallest for the rRNN which actually had learnt this specific walk, see also Table 1. 

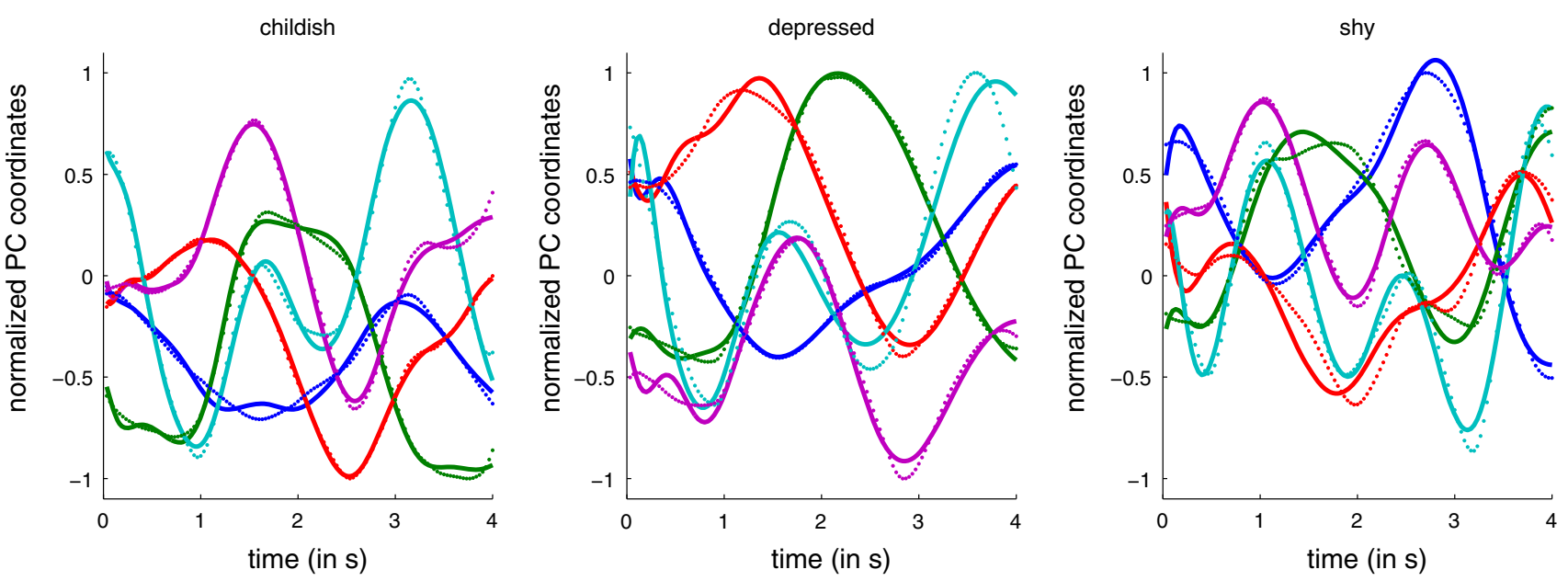

Fig. 3 Dynamics of three different human walks and model fits in principle component space (five components). Dotted lines original dynamics, solid lines trajectories generated by a gRNN after learning. While

the fit between data and its gRNN replications was not perfect, it was sufficiently close such that the gRNN was an appropriate generative model for recognition (see Fig. 5)
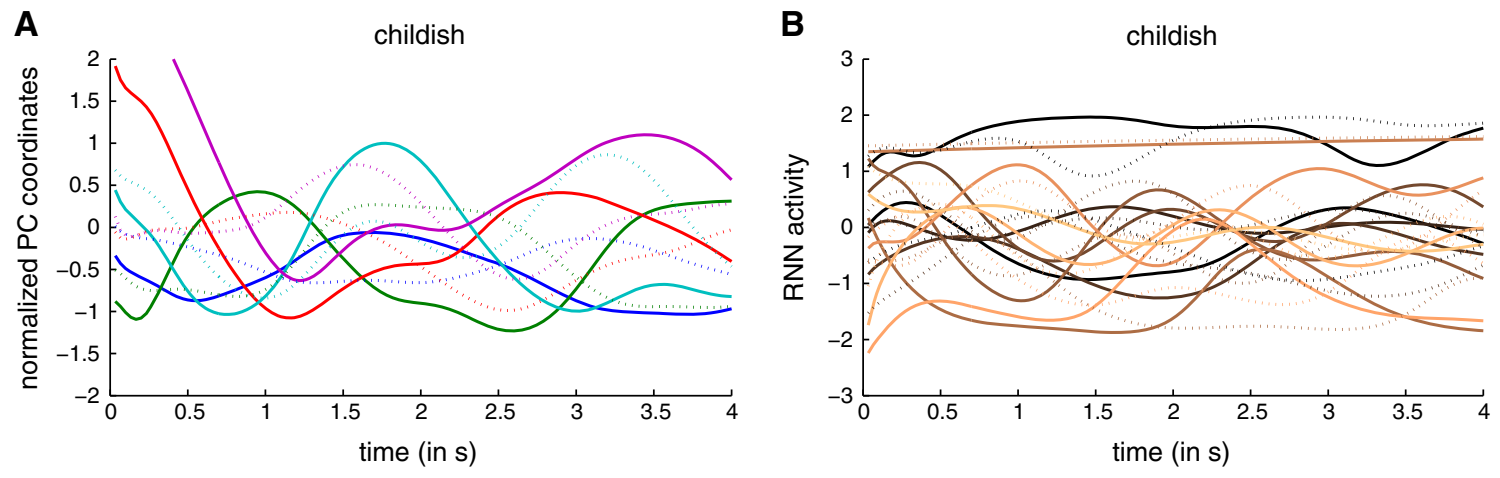

Fig. 4 Influence of initial hidden states on dynamics of generative RNN. Shown are trajectories of sensory (a) and hidden (b) states of the generative RNN for two different initial hidden states. Dotted trajectories resulted from initial hidden states used during learn-

ing $\left(\mathbf{x}(0)^{l}\right)$, while solid trajectories resulted from random initial hidden states $\left(\mathbf{x}(0)^{r}\right)$. In this example, we used the RNN parameters learnt for walk 1 (childish), but results are qualitatively similar for other RNN parameters

The prediction errors on observations showed this effect as well, although not as clearly (Fig. 6a-c).

We also investigated the effect of learning on the accumulated prediction errors by comparing the prediction errors of the learnt rRNNs with those of random rRNNs. We generated 30 random rRNNs by drawing random parameters $\mathbf{W}, \mathbf{V}$ and $\mathbf{k}$ while using the same connectivity constraints as for the gRNNs which were used for learning the walks. The accumulated prediction errors for the random rRNNs, thus, give an estimate for the total amount of prediction error expected in a random rRNN, i.e. without learning. As expected, the prediction errors of random rRNNs were always higher than those of the rRNNs with learnt parameters (see Table 1). Furthermore, for nonlearnt stimuli, the learnt rRNNs often produced larger prediction errors than random rRNNs. This indicates that learning a specific walk restricts the dynamic repertoire of an rRNN. We conclude that the learning

procedure resulted in rRNNs which were suited to discriminate the walks.

In an additional experiment, we concatenated data from all the three walks into a single sequence to simulate online recognition of the three walks, see Fig. 7. The resulting saccade-like, abrupt transitions between walking styles led to a transient increase in prediction errors correctly signalling a discrepancy between predictions and actually observed kinematics. Furthermore, we implemented a simple readout mechanism for dynamic prediction errors using a filter which sums the absolute prediction errors over the last 30 time points and weights recent time points more strongly. This operation smoothes prediction errors temporally and stresses differences that stretch over a similar period as the filter size, see Fig. 7. After each transition, the rRNNs reduced their smoothed prediction errors quickly until the rRNN with parameters learnt for the currently observed walk was the one 

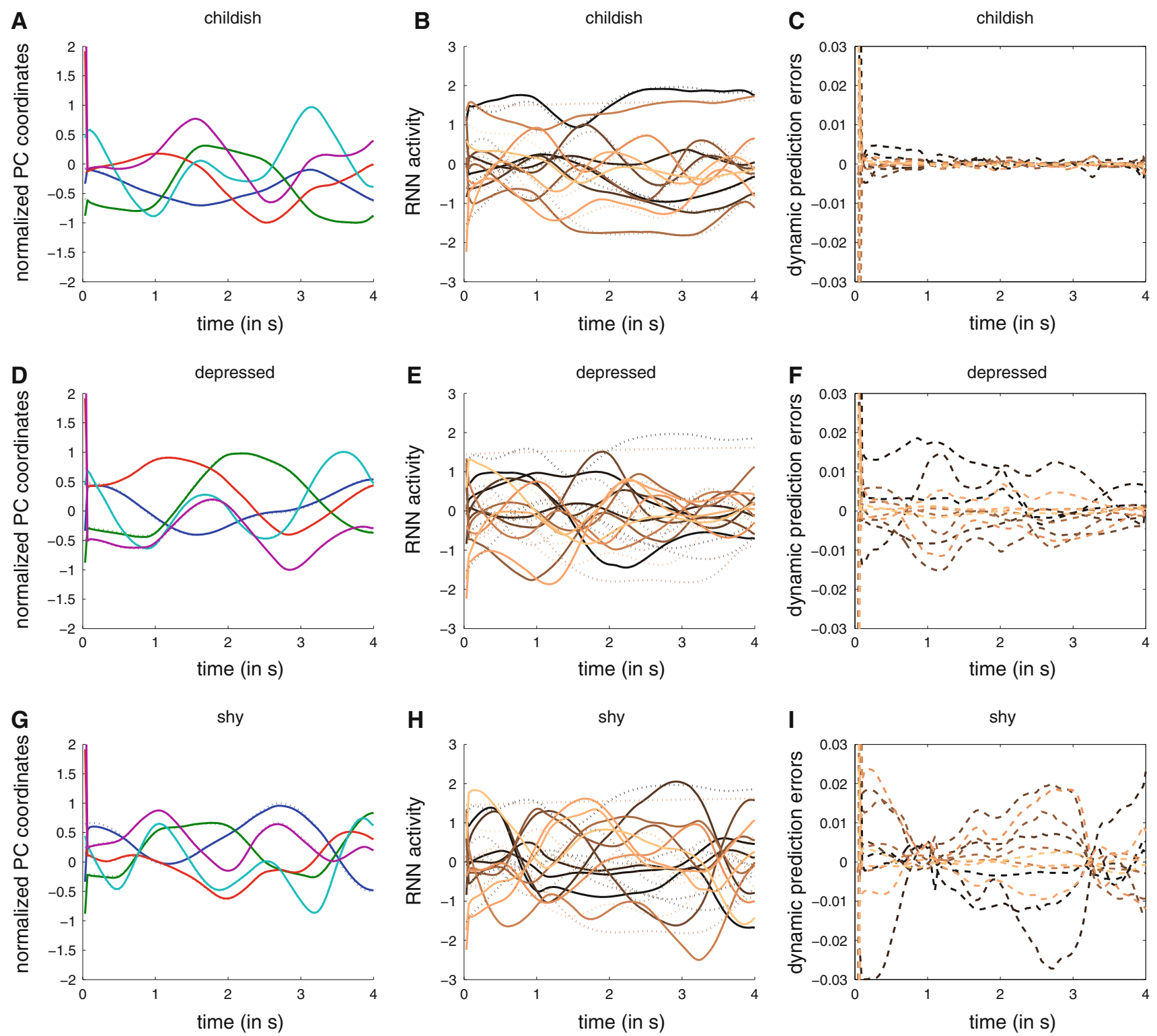

Fig. 5 Result of the three different walks recognized by one of the rRNNs(childish walk). a, d, $\mathbf{g}$ The presented data (dotted lines) and the predicted sensory states (solid lines). b, e, $\mathbf{h}$ The posterior hidden states (solid lines) and, for comparison, the hidden states of the corresponding gRNN when run autonomously from the initial states used during learning (dotted lines, cf. dotted lines in Fig. 4b). c, f, i The dynamic

prediction errors of the hidden states (Eq. 12, note that these prediction errors do not correspond to the difference between solid and dotted lines in the middle panels). The different rows of panels correspond to the different walks which were recognized (from top to bottom: childish, depressed and shy). Prediction errors were markedly lower, when the rRNN recognized the walk it was adapted for ( $\mathbf{c}$ vs $\mathbf{f}, \mathbf{i})$

for which the magnitude of prediction errors was the lowest. This shows that the present approach can be successfully used to recognize a specific walk by choosing the model with the lowest prediction error, after some initial transient has died away.

\subsection{Robustness against noise and initial conditions}

Here, we demonstrate that the recognition scheme is robust to both noise and variations in initial conditions. We repeated

the experiments above for increasing amounts of white observation noise and 12 different, randomly chosen sets of initial conditions, see Fig. 8. We found that the overall magnitude of dynamic prediction errors is proportional to the amount of observation noise. This indicates that observation noise is explained away by prediction errors of both sensory and hidden units. Importantly, the discrimination ability of the three rRNNs is maintained up to moderate amounts of noise, i.e. prediction errors still contained sufficient information to discriminate the three walks. As expected, for large amounts 
Table 1 Absolute prediction errors summed over time points and sensory, or hidden states, respectively

\begin{tabular}{llll}
\hline & Childish & Depressed & Shy \\
\hline Sensory state prediction errors & & & 6.81 \\
rRNN (childish) & $\mathbf{1 . 4 4}$ & 5.58 & 1.53 \\
rRNN (depressed) & 1.69 & $\mathbf{0 . 4 3}$ & $\mathbf{0 . 5 6}$ \\
rRNN (shy) & 2.89 & 2.66 & $4.21(2.57)$ \\
rRNN (random) & $4.37(2.37)$ & $4.30(2.20)$ & 7.38 \\
Hidden state prediction errors & & & 4.05 \\
rRNN (childish) & $\mathbf{0 . 8 5}$ & 5.15 & $\mathbf{1 . 4 8}$ \\
rRNN (depressed) & 5.95 & $\mathbf{1 . 0 6}$ & $4.65(3.03)$ \\
rRNN (shy) & 7.39 & 6.44 & $4.86(3.13)$ \\
rRNN (random) & $4.96(3.04)$ & & \\
\hline
\end{tabular}

Top: sensory state prediction errors. Bottom: dynamic hidden state prediction errors. Each column presents results for each of the three different rRNNs on one of the three data sets childish, depressed and shy. rRNN (random): average accumulated prediction error obtained from 30 random rRNNs (values in parentheses show the minima). rRNN with lowest prediction error on each data set is indicated by bold font. Note that we excluded the first four out of 120 time points from these sums, because the initial transient period otherwise may distort the results
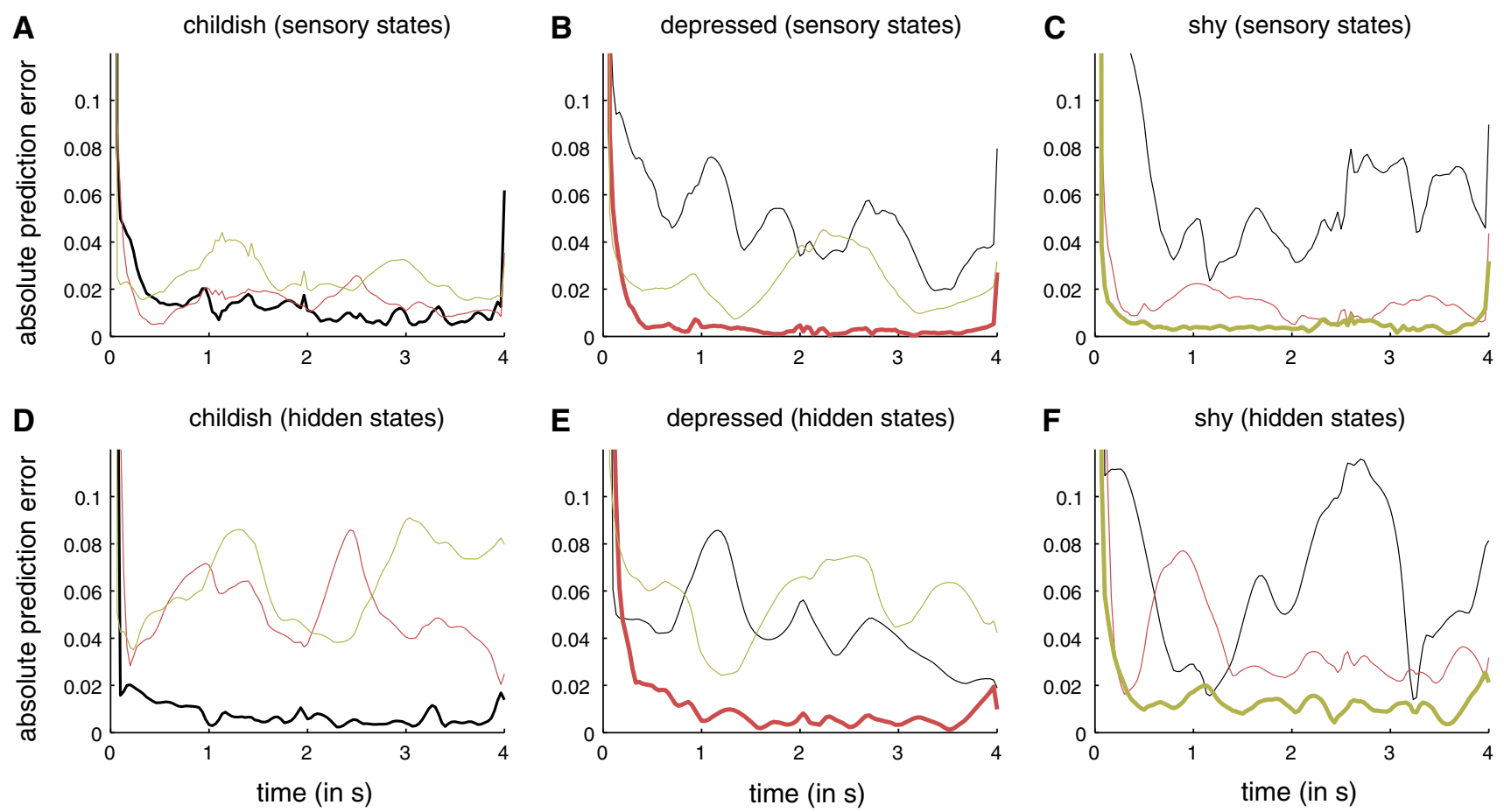

Fig. 6 Comparison of absolute prediction errors. Each panel shows summed (over state dimensions) absolute prediction errors of sensory $(\mathbf{a}-\mathbf{c})$ and hidden $(\mathbf{d}-\mathbf{f}$, Eq. 12) states of the three different rRNNs when data from one of the three different walks were observed. Each rRNN corresponds to one colour (black childish, red depressed, yellow shy). Prediction errors of the rRNN, which has been learnt for the observed type of walk, are indicated by thick lines. of noise, the contribution from observation noise eventually masked the prediction error contributed by the difference in walks. Also, note that the dynamic prediction errors of the learnt rRNNs on their learnt walks (bottom trajectories in the panels of Fig. 8) had very low variability across initial conditions. This means that the rRNN, which was learnt for a specific walk and observes this walk as input, was much less dependent on its initial conditions than the rRNNs learnt on different walks. Yet, the variability of prediction error due to initial conditions within each rRNN was not large enough to influence the result of discrimination of the walks up to moderate amounts of noise. In other words, in our experiments accumulated prediction errors of the rRNN learnt for the current walk were always smaller than those of the other 


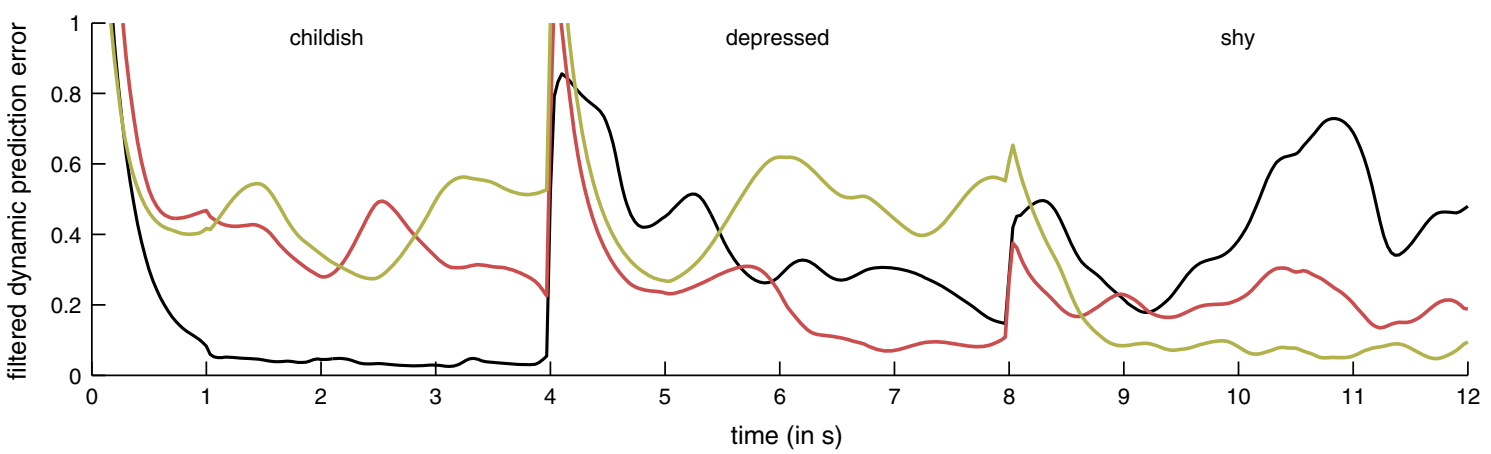

Fig. 7 Filtered dynamic prediction errors. We concatenated sensory input from all walks into a single sequence and inferred the hidden states for all three rRNNs. Shown are temporally smoothed, summed absolute prediction errors for the rRNNs learnt on the childish (black), depressed (red) and shy (yellow) walks.

rRNNs (up to moderate amounts of noise), even when beneficial initial conditions for them led to better than average accumulated prediction errors.

\section{Discussion}

In this paper, we have described the 'recognizing recurrent neural network', which is a RNN where each unit computes both predictions and prediction errors to recognize sensory input in a Bayes-optimal fashion. We derived the update equations of both sensory and hidden units using an approximate Bayesian inference framework for nonlinear dynamical systems, i.e. DEM (Friston et al. 2008). The rRNN approach unifies many important aspects of brain processing such as statistically optimal inference in highly variable and noisy environments, recurrent connections, online recognition of dynamics and quick adaptation to sudden changes in the environment. Therefore, we believe that, compared to conventional RNNs, rRNNs are more appropriate functional models of the computations in recurrently connected units in the brain and may be a useful device to bridge the gap between behaviour-driven models of cognition and neurobiologically motivated models of neuronal ensembles. In particular, rRNNs extend conventional RNNs by (1) providing a Bayesian inference interpretation of the computations done between recurrently connected units and 2) connecting RNNs with the idea of predictive coding which has recently been reappraised in cognitive neuroscience (van Wassenhove et al. 2005; Summerfield et al. 2006; Bar 2009; Friston and Kiebel 2009). This latter point is based on the interpretation of computations in the rRNN as an exchange of prediction and prediction error messages. Consequently, the rRNN approach is a mathematical description of how a predictive coding scheme could be implemented for complex, multidimensional dynamic sensory input.

Recent findings and theoretical considerations show that single neurons (and consequently neuronal ensembles) compute much more complex functions than previously thought
(Sidiropoulou et al. 2006; Spruston 2008; Mel 2008; Pissadaki et al. 2010; Debanne et al. 2011). The general idea is that a single neuron may in principle compute complex, nonlinear and dynamic functions using its spatiotemporal voltage depolarizations and other dynamics like calcium fluctuations (Mel 2008). Although, it is yet unclear how the computation of predictions and prediction errors in the rRNN may map to cellular dynamics, intracellular dynamics and, hence, the dynamics of a neuronal ensemble may have in principle the computational complexity to perform Bayesian decoding of their synaptic input (Denève 2008).

To illustrate that rRNNs may be an interesting model for understanding the brain function of recognition and prediction for naturalistic stimuli, we showed that rRNNs can robustly recognize kinematics as observed with motion capture data. We found that the prediction error computed by an rRNN can be used to recognize and discriminate between different human walking movements in an online fashion. Furthermore, this recognition mechanism is robust against both noise on the observations and variations in the initial state of the rRNN. In other words, rRNNs may be used as functional models for human action observation studies, e.g. Blake and Shiffrar (2007). Here, we have not considered multistable dynamics which is an important phenomenon, particularly for describing coordinated movements (Schöner 1990; Kelso 1995; Mottet and Bootsma 1999; Jirsa and Kelso 2005). Multistability may be harnessed in the present framework by choosing appropriately structured gRNNs, instead of the randomly connected gRNNs used here. For example, the architecture suggested in Perdikis et al. (2011a,b) could be used to build multistable gRNNs. The rRNNs resulting from inversion of such gRNNs would also exhibit the desired multistable dynamics and, importantly, would also exhibit rapid switching between different regimes, if the sensory input shows evidence of such a switch.

The idea to use autonomous RNNs as generative models is not entirely new. In previous work, we have used this approach in system identification where we explained neu- 

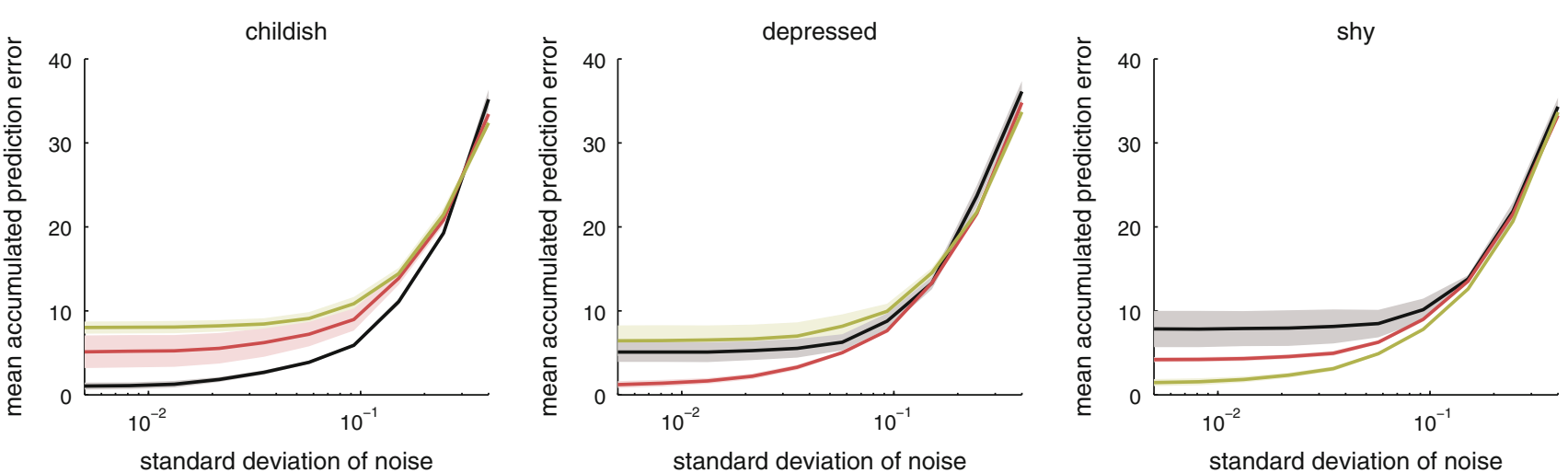

Fig. 8 Dependence of dynamic prediction errors on noise and initial conditions. Each panel shows average sums of absolute prediction errors for the three different rRNNs on one of the walks. The averages are over 12 randomly chosen initial states $\mathbf{x}(0)^{r}$ and shading indicates the region around the mean of twice the standard deviation. The $x$-axis indicates

roimaging data as generated by a network of cortical nodes, called 'Dynamic Causal Modelling' (DCM) (Friston et al. 2003; Kiebel et al. 2006, 2009a). Critically, the equations governing the dynamics of each node took the form of a rate model as in Eq. 3. The difference to the present approach is that DCM uses specific, highly constrained connectivity schemes based on neural mass models and does not allow for errors in the hidden states. Similarly, we used the present approach (Friston et al. 2008) to model recognition of multiscale dynamics (Kiebel et al. 2008, 2009b) where the rRNN generalizes these previous contributions using a more generic generative model (RNNs) and learning of natural stimuli.

To our knowledge, the explicit use of (generic) RNNs as generative models for recognizing dynamic sensory input using online Bayesian inference has not been described before. Both the techniques, Bayesian inference for dynamic stimuli and artificial RNNs have existed in parallel for many years now (Jazwinski 1970; Pearlmutter 1989; Williams and Zipser 1989; Narendra and Parthasarathy 1990). We propose the combination of these two approaches in which RNNs act as dynamic models in a nonlinear, Bayesian filtering framework. Indeed, this idea has already been used implicitly in the field of machine learning and control. For example, Connor et al. (1994) used a related approach in the context of autoregressive models to remove outliers from sequences of discrete states which were represented by the hidden states of a RNN. Also, in dual-extended Kalman filter methods for RNNs (Wan and Nelson 2001), an extended Kalman filter is used to estimate RNN hidden states. However, these contributions focus on the usefulness of Bayesian filtering of RNN states to make conventional RNN learning more robust. Here, we describe the idea that the combination of RNN equations and filter updates can themselves be interpreted as network equations which are better suited for recognizing dynamic stimuli. Therefore, the present approach also goes beyond the standard deviation of independent Gaussian noise added to the principal components of the walks on a log-scale. Note that the exponential increase of prediction errors with noise in the log-plot means that prediction errors depend approximately linear on the observation noise magnitude

previous suggestions of using RNNs as functions approximating the update equations of a nonlinear filter (Parlos et al. 2001), or its output (Ting-Ho Lo 1994). We, thus, provide a novel perspective on the role of RNNs also in possible machine learning applications.

We motivated the present approach by considering the potential functional role of recurrently connected neuronal ensembles in cortical processing. This allowed us to address recognition of arbitrary nonlinear dynamics embedded in multidimensional, continuous stimuli-something that has not been reported with spiking neuron models of neuronal coding in recurrent networks (Rao 2004; Denève et al. 2007; Wilson and Finkel 2009; Boerlin and Denève 2011). In contrast, the 'reservoir computing' approach (Jaeger 2001; Maass et al. 2002; Verstraeten et al. 2007) can recognize the class of stimuli we considered here. The reservoir computing approach has reinvigorated RNN research by establishing that very large networks (hundreds to thousands of units) combined with a simple readout function can be used to learn and recognize both dynamic and static stimuli. However, reservoir computing approaches typically do not adapt the dynamics within the network and rely on the chance probability that, among the many units, there exist some dynamical regimes which are appropriate generative models of the data. Here, we describe an alternative approach and speculate that small networks of 'smart' rRNN units may be sufficient to recognize dynamic stimuli.

Our use of RNNs as generative models of dynamic stimuli requires learning of their parameters ( $\mathbf{W}, \mathbf{V}$ and $\mathbf{k}$ in Eqs. 3, 4). In particular, our results depend on learning the connections between hidden units in the recurrent network $(\mathbf{W})$. This type of learning has been proven to be difficult in the past (Hammer and Steil 2002). While the learning procedure used here was capable of learning a sufficiently good dynamic representation of the present walks alternative learn- 
ing approaches may have to be used to achieve similar performance on other data sets. For example, the different principal component coordinates of our walks had similar time scales (Fig. 3). More complex and longer movements may demand the use of hierarchical models and corresponding learning algorithms (Hinton and Salakhutdinov 2006).

While learning is an important issue with RNNs, we focused on providing a proof-of-principle that the rRNN approach can solve high-level problems such as discriminating visual dynamics in an online fashion. Here, we used a small toy example to show that this is, in principle, possible. Importantly, our results appear to be robust against sub-optimally learnt generative RNNs. This can be seen in Fig. 3, which shows a residual difference between learnt trajectories and the input. In other words, we found that prediction error messages were sufficiently informative even though the sensory input observed by the rRNN deviated slightly from the internally predicted dynamics. We also found robustness of the discrimination against white observation noise, see Fig. 8. It is an open question whether the rRNN approach is also robust against structured variations in human movements, e.g. as induced by a variation of a specific movement. We speculate that such variations require a different generative model, either where multiple movements are embedded in a single gRNN or where one uses a hierarchical gRNN similar to the approach used in Taylor and Hinton (2009). Furthermore, we are currently working on applying the rRNN approach to more complex stimuli than the toy example presented here.

Acknowledgments We thank both anonymous reviewers for the helpful and constructive comments on a previous version of this manuscript.

Open Access This article is distributed under the terms of the Creative Commons Attribution License which permits any use, distribution, and reproduction in any medium, provided the original author(s) and the source are credited.

\section{Appendix A: Bayesian inversion of dynamical models}

In this appendix, we give a high-level description of the Dstep in Friston's dynamic expectation framework (Friston et al. 2008) which leads to Eq. 5 in the main paper.

Appendix B: Generalized coordinates

A major component of Friston's approach to stochastic processes is the redefinition of the time-dependent variables in generalized coordinates of motion. For example, one replaces $\mathbf{x}(t)$ with

$\tilde{\mathbf{x}}(t)=\left[\mathbf{x}(t)^{T}, \frac{\partial \mathbf{x}(t)^{T}}{\partial t}, \frac{\partial^{2} \mathbf{x}(t)^{T}}{\partial t^{2}}, \ldots\right]^{T}$.

and obtains for the probabilistic form of Eq. 3 (dropping the dependence on $t$ for simplicity of writing)

$$
\begin{aligned}
\dot{\mathbf{x}} & =\mathbf{x}^{\prime}=f(\mathbf{x})+\epsilon^{x} \\
\frac{\partial^{2} \mathbf{x}}{\partial t^{2}} & =\mathbf{x}^{\prime \prime}=\frac{\partial f}{\partial \mathbf{x}} \mathbf{x}^{\prime}+\epsilon^{\prime x} \\
\frac{\partial^{3} \mathbf{x}}{\partial t^{3}} & =\mathbf{x}^{\prime \prime \prime}=\frac{\partial f}{\partial \mathbf{x}} \mathbf{x}^{\prime \prime}+\epsilon^{\prime \prime} x
\end{aligned}
$$

Note that it is assumed that $f$ is locally linear around $\mathbf{x}(t)$ and that differently from the usual stochastic process models dependencies between noise variables across time are allowed, i.e. it is assumed that the noise at two close points in time correlates and that the noise process $\epsilon^{x}(t)$ is differentiable sufficiently many times. Generalized coordinates of motion time-dependent variables encode not only a state at the current time but also additionally the future path of states. This is seen when we consider how the continuous representation here can be mapped onto a discrete sequence of $N$ future observations $\mathbf{y}=\left[y_{1}, \ldots, y_{N}\right]^{T}$ (for simplicity we here show only a single observed variable, i.e. $D=1$ )

$y_{i}=\sum_{j=1}^{n} \frac{\tilde{y}_{j}(t)}{(j-1) !}(i-t)^{j-1}$,

where $n$ is the highest order of motion considered. We assume that $i=1, \ldots, N$ are the times starting from $t$ at which the data have been sampled. This formula represents a Taylor series approximation making use of the derivatives in $\tilde{\mathbf{y}}$. Friston et al. (2008) have shown that the variance of the noise process quickly becomes very large for high-order motions such that only a small number $n$ of generalized coordinates and data points need to be taken into account at any time point. One also needs to translate discrete data samples into generalized coordinates of motion. This can be done using the inverse operation of Eq. 16. Rewriting Eq. 16 in matrix form gives

$$
\begin{aligned}
& \mathbf{y}=\mathbf{E} \tilde{\mathbf{y}} \quad e_{i j}=\frac{(i-t)^{j-1}}{(j-1) !} \\
& i \in\{1, \ldots, N\}, \quad j \in\{1, \ldots, n\} .
\end{aligned}
$$

If $N=n, \mathbf{E}$ is invertible and one obtains $\tilde{\mathbf{y}}(t)=\mathbf{E}^{-1} \mathbf{y}$. The resulting $\tilde{\mathbf{y}}(t)$ is then used to compute the likelihood of the data at $t$ and make inference over the hidden RNN states $\tilde{\mathbf{x}}(t)$ as described below.

Dynamic approximation of the posterior mode

In generalized coordinates Eqs. 3 and 4 become

$\tilde{\mathbf{y}}=\tilde{\mathbf{g}}+\tilde{\epsilon}_{y} \quad \mathbf{D} \tilde{\mathbf{x}}=\tilde{\mathbf{f}}+\tilde{\epsilon}_{x}$,

where $\mathbf{D}$ is a matrix differentiation operator which shifts coordinates upwards by one element, $\tilde{\mathbf{f}}=\left[\mathbf{f}^{T}, \mathbf{f}^{\prime}, \mathbf{f}^{\prime \prime} T, \ldots\right]^{T}$ and $\tilde{\mathbf{g}}=\left[\mathbf{g}^{T}, \mathbf{g}^{\prime}, \mathbf{g}^{\prime \prime} T, \ldots\right]^{T}$ are the predicted generalized states and observations, respectively, with $\mathbf{f}^{\prime}=\frac{\partial f}{\partial \mathbf{x}} \mathbf{x}^{\prime}$ and $\mathbf{g}^{\prime}=\mathbf{V} \mathbf{x}^{\prime}$ (analogously for higher order terms $\mathbf{f}^{\prime \prime}, \ldots$. ). Because $\tilde{\mathbf{g}}$ is linear here, one can write $\tilde{\mathbf{g}}=(\mathbf{I} \otimes \mathbf{V}) \tilde{\mathbf{x}}$, where 
Table 2 Online algorithm for finding the approximate posterior of RNN states initialize $\tilde{\mu}(0)$

FOR $t=1: N$

1) compute predictions $\tilde{\mathbf{g}}(\tilde{\mu})$ and $\tilde{\mathbf{f}}(\tilde{\mu})$ from previous $\tilde{\mu}(t-\Delta t)$

2 ) find $\tilde{\mathbf{y}}$ based on $n$ data points closest to $t$ using Eq. 17

3 ) compute gradients of $V(\tilde{\mu})$ using predictions, $\mathbf{D} \tilde{\mu}(t-\Delta t)$ and $\tilde{\mathbf{y}}$

4) numerically integrate Eq. 23 to get new $\tilde{\mu}(t)$

END $\otimes$ denotes the Kronecker product and $\mathbf{I} \in \mathbb{R}^{n \times n}$. Based on these equations, the log-likelihood of the observations $\tilde{\mathbf{y}}(t)$ is defined as

$$
\begin{aligned}
\mathcal{L}(t) & =\log p(\tilde{\mathbf{y}} \mid \theta) \\
& =\log \int p(\tilde{\mathbf{y}}, \tilde{\mathbf{x}} \mid \theta) \mathrm{d} \tilde{\mathbf{x}} \\
& =\log \int p(\tilde{\mathbf{y}} \mid \tilde{\mathbf{x}}, \theta) p(\tilde{\mathbf{x}} \mid \tilde{\mathbf{f}}, \theta) \mathrm{d} \tilde{\mathbf{x}},
\end{aligned}
$$

where $\theta$ is a placeholder for all parameters in the model. Notice that $p(\tilde{\mathbf{f}}(\tilde{\mathbf{x}}) \mid \mathbf{Y})$, where $\mathbf{Y}$ represents previously seen data, has been approximated as $\delta(\tilde{\mathbf{f}}(\tilde{\mu}))$ - a Dirac delta function at $\tilde{\mathbf{f}}$ evaluated at the previous posterior mode $\tilde{\mu}$ (see below). This means that only the mode is propagated through the dynamics, but not its uncertainty. Friston et al. (2008) then introduce a variational density $q(\tilde{\mathbf{x}})$ (ignoring the density over parameters as learning is not our objective) and make use of Jensen's inequality to obtain

$\mathcal{L}(t) \geq \int q(\tilde{\mathbf{x}}) \log \frac{p(\tilde{\mathbf{y}}, \tilde{\mathbf{x}} \mid \theta)}{q(\tilde{\mathbf{x}})} d \tilde{\mathbf{x}}=\mathcal{F}(q, t)$,

where $\mathcal{F}(q, t)$ is the free energy which is a lower bound on the $\log$-likelihood. The aim is to find $q$ such that $\mathcal{L}(t)=\mathcal{F}(q, t)$. In other words, one maximizes $\mathcal{F}(q, t)$ with respect to $q$. This is equivalent to minimizing $K L[q(\tilde{\mathbf{x}}) \| p(\tilde{\mathbf{x}} \mid \tilde{\mathbf{y}}, \theta)]$, the KLdivergence between variational density and true posterior, i.e. after optimization $q$ is an approximation of the posterior density over RNN states. In particular, it can be shown (Ghahramani and Beal 2001; Friston et al. 2008) that the $q$ maximizing $\mathcal{F}(q, t)$ is equal to

$$
\begin{aligned}
q(\tilde{\mathbf{x}}) & =\frac{1}{Z} \exp (V(\tilde{\mathbf{x}})) \\
& =\frac{1}{Z} \exp (\log p(\tilde{\mathbf{y}}, \tilde{\mathbf{x}} \mid \theta)) \\
& =p(\tilde{\mathbf{x}} \mid \tilde{\mathbf{y}}, \theta),
\end{aligned}
$$

where $V(\tilde{\mathbf{x}})$ is called the variational energy. While this equation appears to be a trivial statement, the formulation of $q$ in this way lets us recognize (Friston et al. 2008) that $q$ also is the density defined by a set of stochastically moving particles at their stationary solution where the movement of a single particle is given by $\dot{\tilde{\mathbf{z}}}=\frac{\partial V(\tilde{\mathbf{z}})}{\partial \tilde{\mathbf{z}}}+\Gamma(t)=\frac{\partial \log p(\tilde{\mathbf{y}}, \tilde{\mathbf{z}} \mid \theta)}{\partial \tilde{\mathbf{z}}}+\Gamma(t)$

and $\Gamma(t)$ is a random fluctuation. Using this relationship one can find $q$ using Monte Carlo simulation as we can compute the partial derivative of $\log p(\tilde{\mathbf{y}}, \tilde{\mathbf{z}} \mid \theta)$. However, Friston et al. (2008) simplified this further. In particular, a single particle in generalized coordinates with motion

$\dot{\tilde{\mathbf{z}}}=\kappa \frac{\partial V(\tilde{\mathbf{z}})}{\partial \tilde{\mathbf{z}}}+\mathbf{D} \tilde{\mathbf{z}}$

will converge to the mode $\tilde{\mu}$ of $V$, which is also the mode of the posterior, at a rate proportional to the constant $\kappa$ (Friston et al. 2008). Given the mode $\tilde{\mu}$, Friston et al. (2008) use a Laplace approximation for the posterior where $q \sim \mathcal{N}(\tilde{\mu}, \tilde{\Sigma})$ is defined to be Gaussian and the covariance $\tilde{\Sigma}$ is found as

$\tilde{\Sigma}^{-1}=\tilde{\Pi}=-\left.\frac{\partial^{2} \log p(\tilde{\mathbf{y}}, \tilde{\mathbf{x}} \mid \theta)}{\partial \tilde{\mathbf{x}} \partial \tilde{\mathbf{x}}}\right|_{\tilde{\mathbf{x}}=\tilde{\mu}}$.

This is the inverse of the negative curvature of the posterior evaluated at the mode $\tilde{\mu}$. This completes the derivation of the approximate posterior over RNN states.

Under the approximations made and given the linearity of $g$ one can identify the posterior $p(\tilde{\tilde{\mathbf{x}}} \mid \tilde{\mathbf{y}}, \theta)$ as being Gaussian exploiting that $p(\tilde{\mathbf{y}} \mid \tilde{\mathbf{x}}, \theta)$ and $p(\tilde{\mathbf{x}} \mid \tilde{\mathbf{f}}, \theta)$ are Gaussian. In this case, the Laplace approximation is exact. Nevertheless, we retained Friston's more general form which is also valid for nonlinear $g$. More importantly, this motivates the dynamic form of estimating the posterior mode in Eq. 23 which allows us to extend the static result above to the dynamic case. In particular, note that all results above were obtained for only a single time point $t$. However, it can be shown (Friston et al. 2008) that the path integral of the free energy is maximized, if Eq. 21 holds for all $t$. Naively, this means that one has to integrate the motion of the particle in Eq. 23 until it converges to $\tilde{\mu}(t)$ for each $t$. However, if the particle converges faster onto $\tilde{\mu}(t)$ than $\tilde{\mu}$ moves itself, a condition which can be ensured by choosing an appropriate rate constant $\kappa$, we will be able to track the motion of $\tilde{\mu}$ with a single particle and the dynamics given by Eq. 23. Intuitively, the representation in generalized coordinates of motion here helps to converge to a mode which better represents the data as it also takes the local motion (velocity, acceleration, etc.) of the mode into account. 
For the purpose of this paper, we ignored the approximated covariance and only concentrated on the posterior mode and the corresponding prediction errors. A summary of the resulting algorithm is shown in Table 2. We were able to ignore the covariance, because we assumed network parameters to be fixed during inversion. However, in the full DEM-framework these covariances are needed for the computation of parameter updates.

\section{Appendix B: Learning of RNN parameters}

We want to adapt the RNN parameters $\mathbf{W}, \mathbf{V}, \mathbf{k}$ such that the observations generated by the RNN defined in Eqs. 3 and 4 fit the data. We mainly follow the approach underlying DCM (Friston et al. 2003; Kiebel et al. 2009a) which is detailed in Friston (2002) and Friston et al. (2002). This entails an iterative approximation of the parameter posterior based on a first-order Taylor expansion of an observation function $\operatorname{vec}(\mathbf{Y})=h(\theta)$ which represents the underlying dynamical system. Here, $\mathbf{Y} \in \mathbb{R}^{N \times D}$ contains the observations at all $N$ time points and $\theta=\left[\operatorname{vec}(\mathbf{W})^{T}, \operatorname{vec}(\mathbf{V})^{T}, \mathbf{k}^{T}\right]^{T}$. The RNN states are enclosed in $h(\theta)$, because the dynamics is assumed to be noise free, i.e. deterministic. Both parameter likelihood and prior are assumed to be Gaussian so that the following gradients of the $\log$-posterior $\mathcal{L}=\log p(\theta \mid \mathbf{Y}) \propto$ $\log p(\mathbf{Y} \mid \theta) p(\theta)$ are obtained (cf., Friston 2002, Eq. 17)

$$
\begin{aligned}
\frac{\partial \mathcal{L}}{\partial \theta} & =\mathbf{J}^{T} \mathbf{C}_{y}^{-1} \mathbf{r}+\mathbf{C}_{\theta}^{-1}\left(\mu_{\theta}-\hat{\theta}^{(i)}\right) \\
\frac{\partial^{2} \mathcal{L}}{\partial \theta^{2}} & \approx \mathbf{J}^{T} \mathbf{C}_{y}^{-1} \mathbf{J}+\mathbf{C}_{\theta}^{-1} .
\end{aligned}
$$

We use these in a numerical integration scheme for nonlinear dynamical systems to obtain an update of the parameters $\mathrm{d} \theta$ based on the model $\mathrm{d} \theta / \mathrm{d} t=\partial \mathcal{L} / \partial \theta$ and $\hat{\theta}^{(i+1)}=\hat{\theta}^{(i)}+$ $\mathrm{d} \theta$. Here, $\hat{\theta}^{(i)}$ is the maximum a posteriori estimate of the parameters in iteration $i,[\mathbf{J}]_{j k}=\partial\left[h\left(\hat{\theta}^{(i)}\right)\right]_{j} / \partial \theta_{k}$ is the Jacobian of $h$ evaluated at $\hat{\theta}^{(i)}, \mathbf{C}_{y}$ is the covariance of the observations and $\mu_{\theta}$ and $\mathbf{C}_{\theta}$ are the prior mean and covariance of the parameters, respectively. Finally, $\mathbf{r}=\operatorname{vec}(\mathbf{Y})-h\left(\hat{\theta}^{(i)}\right)$ are the residuals of the data not explained by the predictions $h\left(\hat{\theta}^{(i)}\right)$ which are equivalent to the observation prediction errors $\varepsilon_{y}$ described in the main text. In each iteration, one obtains the predictions $h\left(\hat{\theta}^{(i)}\right)$ by numerical integration of the RNN dynamics and the Jacobian $\mathbf{J}$ using numerical differentiation of $h\left(\hat{\theta}^{(i)}\right)$.

In our experiments, we divided learning into two phasesan initial phase in which we adapted parameters only on local chunks of the data and a final phase in which we used the complete data. We found that the first phase helped to find a better initialization of $\hat{\theta}$ for the optimization on the whole data set in the second phase of learning. In the first phase, we split the data into seven overlapping, equal size chunks and ran two passes through all chunks where we ran only two iterations of the update procedure described above per chunk and pass. In the second phase, we ran 25 iterations with a fast, approximate numerical integration scheme for $h$ and subsequently another 4 iterations with a slower, but more accurate scheme. While our choices for the number of chunks, passes and iterations led to good results, we expect that many other values may be chosen equivalently.

Embedded in each iteration there is also an expectation maximization (EM)-like update of hyperparameter $\lambda$ which determines the amount of noise on the observations $\mathbf{C}_{y}=\mathrm{e}^{\lambda} \mathbf{I}$ during learning. We refer the reader (Friston 2002; Friston et al. 2002) for details. $\lambda$ was initialized as -32 . The hyperprior for $\lambda$ was Gaussian $\lambda \sim \mathcal{N}\left(-\log \left(\bar{s}^{2}\right), 1 / 8\right)$ where $\bar{s}^{2}$ is the average variance of the observation variables in the data.

We initialized the parameters contained in $\theta^{(0)}$ as follows. The elements of $\mathbf{k}^{(0)}$ were chosen uniformly in the interval [1/8, 3/8]. Randomly chosen $2 / 3$ of all elements in $\mathbf{W}^{(0)}$ were fixed at 0 , the remaining were drawn randomly from a standard normal distribution. Furthermore, following Jaeger et al. (2007), we scaled the resulting matrix by $\mathbf{W}=1 /(0.95 \delta) \mathbf{W}^{(0)}$ to bring the initial RNN dynamics to a useful dynamical regime. $\delta$ here is the largest absolute eigenvalue of the matrix $\left[\bar{k} \mathbf{W}^{(0)}-(1-\bar{k} a) \mathbf{I}\right]$ where $a$ is the leakage (cf. Eq. 1) and $\bar{k}=1 / 4$ is the expected value of $k_{i}$ for any $i$. The initial states $x_{j}(0)^{l}$ were chosen uniformly in [-2, 2] for all $j$. We then found $\mathbf{V}^{(0)}$ as the solution to the underdetermined system of equations $\mathbf{y}(0)=\mathbf{V}^{(0)} \mathbf{x}(0)^{l}$ using Matlab's backslash operator, i.e. we found the least squares solution for $\mathbf{V}^{(0)}$ with most elements of $\mathbf{V}^{(0)}$ equal to zero. A randomly chosen subset of these zero elements were also fixed during learning. The number of fixed elements was $1 / 3$ of the total number of elements.

In the initial learning phase, we set the mean of the parameter prior to the described initialization of the parameters $\mu_{\theta}=\theta^{(0)}$. In the subsequent learning phase, we set $\mu_{\theta}$ to the result of the first phase. The covariances of the prior parameter distribution were chosen to be diagonal, but also differed in the two phases of learning. In the initial phase, we set the variances associated with the elements of $\mathbf{W}$ to $1.6 \cdot 10^{5}$ while we set the variances for $\mathbf{V}$ and $\mathbf{k}$ to 0.018 and 0.135 , respectively. This enforced particularly the adaptation of the dynamical parameters. For learning on the full data set, we chose these variances to be $7.389,1$ and 1 for $\mathbf{W}, \mathbf{V}$ and $\mathbf{k}$, respectively.

\section{Appendix C: Prior covariances}

For the rRNN the prior covariances, $\tilde{\Sigma}_{y}$ and $\tilde{\Sigma}_{x}$, modulate the size of updates of the posterior (cf. Eqs. 10, 9) and influence the result of the Bayesian inversion. Intuitively, for large 
prior (co-)variances, i.e. a large amount of a priori expected noise, smaller updates are made and larger prediction errors are tolerated. The amount of noise here has to be seen in comparison to the variance of the unperturbed states of the units in the gRNN. For the sensory states, this corresponds to the variance of the movement data. The standard deviation of the sensory states across all walks averaged over the five input dimensions was 0.38 while the standard deviation of the corresponding changes in hidden states averaged over the 12 hidden units was 0.04 . In our simulations, we assumed isotropic prior noise and correspondingly chose covariances of the form $\Sigma_{y}=\sigma_{y}^{2} \mathbf{I},{ }^{2}$ where $\mathbf{I}$ is the identity matrix and $\sigma_{y}$ is our choice of standard deviation. We chose $\sigma_{y}=0.3$ and $\sigma_{x}=0.1$ for sensory and hidden states, respectively. This means that we tolerated only relatively small prediction errors on sensory states while allowing for relatively larger prediction errors on changes of hidden states. This choice implements the natural prior belief that the variability of walk observations is mainly determined by the variability of the underlying dynamics.

\section{References}

Archambeau C, Opper M, Shen Y, Cornford D, Shawe-Taylor J (2008) Variational inference for diffusion processes. In: Platt J, Koller D, Singer Y, Roweis SAdvances in neural information processing systems. MIT Press, Cambridge, pp 17-24

Bar M (2009) The proactive brain: memory for predictions. Philos Trans R Soc Lond B 364(1521): 1235-1243. doi:10.1098/rstb. 2008.0310

Blake R, Shiffrar M (2007) Perception of human motion. Annu Rev Psychol 58: 47-73. doi:10.1146/annurev.psych.57.102904. 190152

Boerlin M, Denève S (2011) Spike-based population coding and working memory. PLoS Comput Biol 7(2):e1001-080. doi:10.1371/ journal.pcbi. 1001080

Buonomano DV, Maass W (2009) State-dependent computations: spatiotemporal processing in cortical networks. Nat Rev Neurosci 10(2): 113-125. doi:10.1038/nrn2558

Cessac B, Samuelides M (2007) From neuron to neural networks dynamics. Eur Phys J 142: 7-88. doi:10.1140/epjst/ e2007-00058-2

Connor JT, Martin RD, Atlas LE (1994) Recurrent neural networks and robust time series prediction. IEEE Trans Neural Netw 5(2): 240254. doi:10.1109/72.279188

Daunizeau J, Friston K, Kiebel S (2009) Variational Bayesian identification and prediction of stochastic nonlinear dynamic causal models. Physica D 238(21): 2089-2118. doi:10.1016/j.physd.2009. 08.002

Debanne D, Campanac E, Bialowas A, Carlier E, Alcaraz G (2011) Axon physiology. Physiol Rev 91(2): 555-602. doi:0. 1152/physrev.00048.2009

Denève S (2008) Bayesian spiking neurons i inference. Neural Comput 20(1): 91-117. doi:10.1162/neco.2008.20.1.91

\footnotetext{
${ }^{2}$ To see how these standard covariance matrices are translated into generalized coordinates, we refer the reader to Friston et al. (2008, p. 860).
}

Denève S, Duhamel JR, Pouget A (2007) Optimal sensorimotor integration in recurrent cortical networks a neural implementation of Kalman filters. J Neurosci 27(21): 5744-5756. doi:10.1523/ JNEUROSCI.3985-06.2007

Doucet A, Tadić V (2003) Parameter estimation in general state-space models using particle methods. Ann Inst Stat Math 55: 409-422. doi:10.1007/BF02530508

Doucet A, de Freitas N, Gordon N (eds) (2001) Sequential Monte Carlo Methods in Practice. Springer, Berlin

Elman JL (1990) Finding structure in time. Cogn Sci 14(2): 179-211. doi:10.1207/s15516709 $\operatorname{cog} 1402 \_1$

Friston KJ (2002) Bayesian estimation of dynamical systems an application to fMRI. NeuroImage 16(2): 513-530. doi:10.1006/nimg. 2001.1044

Friston K, Kiebel S (2009) Predictive coding under the free-energy principle. Philos Trans R Soc Lond B 364(1521): 1211-1221. doi:10. 1098/rstb.2008.0300

Friston KJ, Penny W, Phillips C, Kiebel S, Hinton G, Ashburner J (2002) Classical and bayesian inference in neuroimaging theory. NeuroImage 16(2): 465-483. doi:10.1006/nimg.2002.1090

Friston KJ, Harrison L, Penny W (2003) Dynamic causal modelling. NeuroImage 19(4):1273-1302

Friston K, Trujillo-Barreto N, Daunizeau J (2008) DEM A variational treatment of dynamic systems. NeuroImage 41(3): 849-885. doi:10.1016/j.neuroimage.2008.02.054

Friston K, Stephan K, Li B, Daunizeau J (2010) Generalised filtering. Math Probl Eng. Article ID 621, 670. doi:10.1155/2010/621670

Ghahramani Z, Beal MJ (2001) Propagation algorithms for variational bayesian learning. In: Leen T, Dietterich T, Tresp V (eds) Advances in neural information processing systems, vol 13, MIT Press, Cambridge, pp 507-513

Hamker FH (2005) The reentry hypothesis the putative interaction of the frontal eye field ventrolateral prefrontal cortex and areas v4 it for attention and eye movement. Cereb Cortex 15(4): 431-447. doi:10.1093/cercor/bhh146

Hammer B, Steil JJ (2002) Tutorial Perspectives on learning with rnns. In: Proceedings of European symposium on artificial neural networks (ESANN) d-side publi, pp 357-368

Hinton GE, Salakhutdinov RR (2006) Reducing the dimensionality of data with neural networks. Science 313(5786): 504-507. doi:10. $1126 /$ science. 1127647

Jaeger H (2001) The "echo state" approach to analysing and training recurrent neural networks. GMD Report 148, German National Research Center for Information Technology

Jaeger H, Lukosevicius M, Popovici D, Siewert U (2007) Optimization and applications of echo state networks with leaky-integrator neurons. Neural Netw 20(3): 335-352. doi:10.1016/j.neunet.2007. 04.016

Jazwinski AH (1970) Stochastic processes and filtering theory. Academic Press, New York

Jirsa VK, Kelso JAS (2005) The excitator as a minimal model for the coordination dynamics of discrete and rhythmic movement generation. J Mot Behav 37(1): 35-51. doi:10.3200/JMBR.37.1.35-51

Kantas N, Doucet A, Singh SS, Maciejowski JM (2009) Overview of sequential monte carlo methods for parameter estimation on general state space models. In: Proceedings of the 15th IFAC symposium on system identification (SYSID), Saint-Malo, France

Kelso JAS (1995) Dynamic patterns: the self-organization of brain and behavior. MIT Press, Cambridge

Kiebel SJ, David O, Friston KJ (2006) Dynamic causal modelling of evoked responses in EEG/MEG with lead field parameterization. NeuroImage 30(4): 1273-1284. doi:10.1016/j.neuroimage.2005. 12.055

Kiebel SJ, Daunizeau J, Friston KJ (2008) A hierarchy of time-scales and the brain. PLoS Comput Biol 4(11): e1000-209. doi:10.1371/ journal.pcbi.1000209 
Kiebel SJ, Garrido MI, Moran R, Chen CC, Friston KJ (2009a) Dynamic causal modeling for eeg and meg. Hum Brain Mapp 30(6): 1866-1876. doi:10.1002/hbm.20775

Kiebel SJ, von Kriegstein K, Daunizeau J, Friston KJ (2009b) Recognizing sequences of sequences. PLoS Comput Biol 5(8): e1000464. doi:10.1371/journal.pcbi.1000464

Lazar A, Pipa G, Triesch J (2009) Sorn a self-organizing recurrent neural network. Front Comput Neurosci 3:23. doi:10.3389/neuro.10. 023.2009

Legenstein R, Maass W (2007) What makes a dynamical system computationally powerful? In: Haykin S, Principe JC, Sejnowski TJ, McWhirter JG (eds) New directions in statistical signal processing: from systems to brains, MIT Press, Cambridge, pp 127-154

Maass W, Natschlger T, Markram H (2002) Real-time computing without stable states a new framework for neural computation based on perturbations. Neural Comput 14(11): 2531-2560. doi:10.1162/ 089976602760407955

Mel BW (2008) Why have dendrites? a computational perspective, Chap. 16. In: Stuart G, Spruston N , Häusser M (eds) Dendrites, 2nd edn. Oxford University Press, Oxford

Miller EK, Cohen JD (2001) An integrative theory of prefrontal cortex function. Annu Rev Neurosci 24: 167-202. doi:10.1146/annurev. neuro.24.1.167

Mottet D, Bootsma RJ (1999) The dynamics of goal-directed rhythmical aiming. Biol Cybern 80(4): 235-245. doi:10.1007/ s004220050521

Mumford D (1996) Pattern theory: a unifying perspective. In: Knill DC Richards W (eds) Perception as Bayesian inference. Cambridge University Press, Cambridge

Narendra K, Parthasarathy K (1990) Identification and control of dynamical systems using neural networks. IEEE Trans Neural Netw 1(1): 4-27. doi:10.1109/72.80202

Natarajan R, Huys QJM, Dayan P, Zemel RS (2008) Encoding and decoding spikes for dynamic stimuli. Neural Comput 20(9): http://www.mitpressjournals.org/doi/pdf/10.1162/neco.2008. 01-07-436 2325-2360. doi:10.1162/neco.2008.01-07-436

Parlos A, Menon S, Atiya A (2001) An algorithmic approach to adaptive state filtering using recurrent neural networks. IEEE Trans Neural Netw 12(6): 1411-1432. doi:10.1109/72.963777

Pearlmutter BA (1989) Learning state space trajectories in recurrent neural networks. Neural Comput 1(2): http://www. mitpressjournals.org/doi/pdf/10.1162/neco.1989.1.2.263 263269. doi:10.1162/neco.1989.1.2.263

Perdikis D, Huys R, Jirsa V (2011a) Complex processes from dynamical architectures with time-scale hierarchy. PLoS One 6(2): e10 589. doi:10.1371/journal.pone.0016589

Perdikis D, Huys R, Jirsa VK (2011b) Time scale hierarchies in the functional organization of complex behaviors. PLoS Comput Biol 7(9): e1002-198. doi:10.1371/journal.pcbi.1002198

Pissadaki EK, Sidiropoulou K, Reczko M, Poirazi P (2010) Encoding of spatio-temporal input characteristics by a ca1 pyramidal neuron model. PLoS Comput Biol 6(12): e1001-038. doi:10.1371/ journal.pcbi.1001038

Poirazi P, Brannon T, Mel BW (2003) Pyramidal neuron as two-layer neural network. Neuron 37(6): http://dx.doi.org 989-999. doi:10. 1016/S0896-6273(03)00149-1

Rabinovich MI, Varona P, Selverston AI, Abarbanel HDI (2006) Dynamical principles in neuroscience. Rev Mod Phys 78(4): 1213-1265. doi:10.1103/RevModPhys.78.1213

Rao RPN (2004) Bayesian computation in recurrent neural circuits. Neural Comput 16(1): 1-38. doi:10.1162/08997660460733976

Rao RP, Ballard DH (1997) Dynamic model of visual recognition predicts neural response properties in the visual cortex. Neural Comput 9(4):721-763
Rao RP, Ballard DH (1999) Predictive coding in the visual cortex: a functional interpretation of some extra-classical receptive-field effects. Nat Neurosci 2(1): 79-87. doi:10.1038/4580

Rodrigues S, Chizhov AV, Marten F, Terry JR (2010) Mappings between a macroscopic neural-mass model and a reduced conductance-based model. Biol Cybern 102(5): 361-371. doi:10.1007/ s00422-010-0372-z

Roweis S, Ghahramani Z (2001) Learning nonlinear dynamical systems using the expectation-maximization algorithm. In Haykin S (ed) Kalman filtering and neural networks. Wiley, New York. doi:10. 1002/0471221546

Schön TB, Wills A, Ninness B (2011) System identification of nonlinear state-space models. Automatica 47(1): 39-49. doi:10.1016/j. automatica.2010.10.013

Schöner G (1990) A dynamic theory of coordination of discrete movement. Biol Cybern 63(4): 257-270. doi:10.1007/BF00203449

Sidiropoulou K, Pissadaki EK, Poirazi P (2006) Inside the brain of a neuron. EMBO Rep 7(9): 886-892. doi:10.1038/sj.embor. 7400789

Sotero RC, Trujillo-Barreto NJ, Iturria-Medina Y, Carbonell F, Jimenez JC (2007) Realistically coupled neural mass models can generate eeg rhythms. Neural Comput 19(2): 478-512. doi:10.1162/neco. 2007.19.2.478

Spruston N (2008) Pyramidal neurons: dendritic structure and synaptic integration. Nat Rev Neurosci 9(3): 206-221. doi:10.1038/ nrn2286

Summerfield C, Egner T, Greene M, Koechlin E, Mangels J, Hirsch $\mathrm{J}$ (2006) Predictive codes for forthcoming perception in the frontal cortex. Science 314(5803): 1311-1314. doi:10.1126/science. 1132028

Taylor GW, Hinton GE (2009) Factored conditional restricted boltzmann machines for modeling motion style. In: Proceedings of the 26th international conference on machine learning (ICML)

Ting-Ho Lo J (1994) Synthetic approach to optimal filtering. IEEE Trans Neural Netw 5(5): 803-811. doi:10.1109/72.317731

Valpola H, Karhunen J (2002) An unsupervised ensemble learning method for nonlinear dynamic state-space models. Neural Comput 14(11): http://www.mitpressjournals.org/doi/ pdf/10.1162/089976602760408017 2647-2692. doi:10.1162/ 089976602760408017

van Wassenhove V, Grant KW, Poeppel D (2005) Visual speech speeds up the neural processing of auditory speech. Proc Natl Acad Sci USA 102(4): 1181-1186. doi:10.1073/pnas.0408949102

Verstraeten D, Schrauwen B, D'Haene M, Stroobandt D (2007) An experimental unification of reservoir computing methods. Neural Netw 20(3): 391-403. doi:10.1016/j.neunet.2007.04.003

Wan EA, van der Merwe R (2001) The unscented Kalman filter. In Haykin S (ed) Kalman Filtering and Neural Networks. Wiley, New York. doi:10.1002/0471221546

Wan EA, Nelson AT (2001) Dual extended Kalman filter methods. In Haykin S (ed) Kalman Filtering and Neural Networks. Wiley, New York. doi:10.1002/0471221546

Williams RJ, Zipser D (1989) A learning algorithm for continually running fully recurrent neural networks. Neural Comput 1(2): http://www.mitpressjournals.org/doi/pdf/10.1162/neco. 1989.1.2.270 270-280. doi:10.1162/neco.1989.1.2.270

Wilson R, Finkel L (2009) A neural implementation of the Kalman filter. In: Bengio Y, Schuurmans D, Lafferty J, Williams CKI, Culotta A (eds) Advances in neural information processing systems, vol 22. MIT Press, Cambridge, pp 2062-2070 